(C) 2020 IEEE. Personal use of this material is permitted. Permission from IEEE must be obtained for all other uses, in any current or future media, including reprinting/republishing this material for advertising or promotional purposes,creating new collective works, for resale or

redistribution to servers or lists, or reuse of any copyrighted component of this work in other works. DOI 10.1109/IGARSS39084.2020.9324044

\title{
OFFSHORE DOPPLER WIND LIDAR ASSESSMENT OF ATMOSPHERIC STABILITY
}

\author{
Araujo da Silva, M.P ${ }^{1 *}$, Student Member, IEEE, Salcedo-Bosch, A. ${ }^{1}$, Gutierrez-Antunano, M. A. ${ }^{1}$, \\ Rocadenbosch, $F^{1,2 *}$, Senior Member, IEEE. \\ ${ }^{1}$ CommSensLab-UPC, Department of Signal Theory and Communications (TSC), Universitat Politècnica de Catalunya (UPC), \\ Barcelona, E-08034, Spain \\ ${ }^{2}$ Institut d'Estudis Espacials de Catalunya (Institute of Space Studies of Catalonia, IEEC), Barcelona, E-08034, Spain \\ *Corresponding authors: marcos.silva@upc.edu, roca@tsc.upc.edu
}

\section{ABSTRACT}

4 This paper tackles atmospheric stability typing using a Zephyr ${ }^{\mathrm{TM}} 300$ offshore Doppler Wind Lidar in the context of its progressive acceptance in the offshore wind-energy industry. The lidar-retrieved wind-shear exponent, which is used as a proxy atmospheric stability, is compared against the wind-shear exponent and the potential temperature gradient both retrieved from reference metmast. A total sample of 4319 measurements is analysed from IJmuiden's test campaign, in the North Sea, from

This work was supported via Spanish Government-European Regional Development Funds project PGC2018-094132-B-I00 and EU H2020 ACTRIS2 (GA 654109). The European Institute of Innovation and Technology (EIT), KIC InnoEnergy project NEPTUNE (Offshore Metocean Data Measuring Equipment and Wind, Wave and Current Analysis and Forecasting Software, call FP7) supported measurements campaigns. CommSensLab is a Maríade-Maeztu Unit of Excellence funded by the Agencia Estatal de Investigación (Spanish National Science Foundation) that also funded the project MDM2016-0600-18-1. Spanish NSF (Ministry of Science, Innovation and Universities; Ministerio de Ciencia, Innovación y Universidades) for doctoral grant PRE2018-086054 hold by Ph.D. student Araújo da Silva M.P. at CommSensLab-UPC. 
8

9

April $1^{\text {st }}$ to $30^{\text {th }}, 2015$. Concerning stability typing, both lidar- and metmast-derived wind-shear indicators overestimated by $4 \%$ and $14 \%$, respectively, the most frequent atmospheric stability case, which was convective (48\% of the cases) according to the potential temperature reference indicator.

Index Terms - Wind lidar, wind shear, atmospheric stability, offshore wind energy

\section{INTRODUCTION}

Europe is one of the regions with higher investment in offshore wind energy over the last decade with UK and Germany standing out [1]. However, offshore wind farms still have higher production costs than onshore ones. Besides, up to now, the atmospheric reference instruments offshore have been costly metmasts. Today, more and more, floating Doppler Wind lidars (DWL) on buoys provide competitively low-cost alternative and offer key benefits to the offshore wind industry such as lower costs of installation and easier re-deployment [2].

The study of atmospheric stability has been a subject of interest to the wind energy industry [3]. Vertical distribution of momentum, wind shear and hub height wind speed are impacted by atmospheric stability which is usually classified as convective, neutral or stable [3], [4]. Fatigue loads in wind turbines, induced by turbulence intensity magnitudes variations, are also related to stability regime. According to atmospheric stability type, turbulence and wind speed can greatly vary affecting the wind turbines power performance [3], [4], [5].

There are different techniques to study atmospheric stability such as the vertical profiles of potential temperature, the Obukhov length, turbulence intensity (TI), and wind shear exponent [5], [6]. Among them, potential temperature is widely recognised as a trustworthy and physically-rooted indicator because it gives information about the dynamics of air parcels with height. The wind-shear parameter is often used in engineering literature and provides a straightforward power-law approximation of the vertical wind profile. Besides, it gives a direct measure of the relative shear (vertical wind speed gradient) and is related to the stress on wind turbine generators [5], [6]. 
The present work proposes to use the floating DWL to estimate the wind shear as a proxy for offshore atmospheric stability classification. This paper is structured as follows: Sect. 2 presents the floating DWL and the IJmuiden test site. Sect. 3 describes the methods used to estimate wind shear and atmospheric stability typing. Sect. 4 discusses consistency of the experimental results when comparing floating DWL EOLOS ${ }^{\mathrm{TM}}$ against IJmuiden metmast, and Sect. 5 gives conclusion remarks.

\section{INSTRUMENTS}

Experimental data was collected at IJmuiden offshore test site, in the North Sea, $85 \mathrm{~km}$ far from the coast of Netherlands (52.848

N, 3.436 E, Fig. 1). The analysed period was from April $1^{\text {st }}$ to $30^{\text {th }}, 2015$ (4319 data records). The lidar buoy assembled a ZephIR $^{\mathrm{TM}} 300 \mathrm{DWL}$, which is a continuous-wave focusable lidar designed for offshore operation. Temporal resolution of the Horizontal Wind Speed (HWS) data is 10 min.

The raw data included the following metmast sensors: (i) six Thies advanced cup anemometers, sited at $27 \mathrm{~m}$ and $58.5 \mathrm{~m}$ (three at each altitude), (ii) three METEK sonic anemometers, at $85 \mathrm{~m}$, (iii) two temperature sensors Vaisala HMP155D at 21 $\mathrm{m}$ and $90 \mathrm{~m}$, and (iv) two air-pressure sensors Vaisala PTB210 at $21 \mathrm{~m}$ and $90 \mathrm{~m}$ [7], [8].

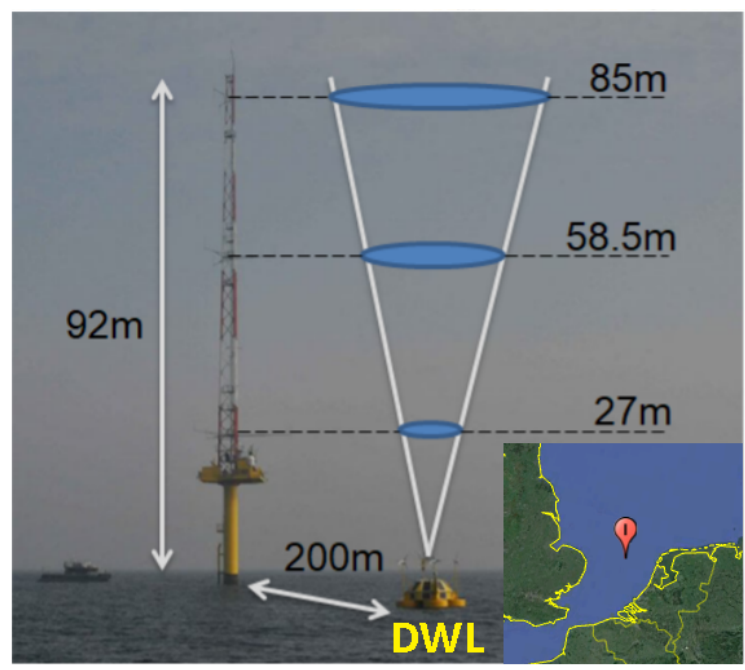

Fig. 1. IJmuiden offshore meteorological test site measurement configuration. 


\section{METHODS}

\subsection{Estimation of the wind-shear parameter}

Both the floating DWL and metmast anemometers were used to independently compute the wind shear exponent by using the

$$
U(z)=U_{R}\left(\frac{z}{z_{R}}\right)^{\alpha},
$$

whe

h

cov

$10-1$

m

$\mathrm{m}$ and $83 \mathrm{~m}$, whereas the metmast wind-shear exponent, $\alpha_{\text {mast }}$, used the HWS measured at $27 \mathrm{~m}, 58.5 \mathrm{~m}$ and $85 \mathrm{~m}$.

\subsection{Atmospheric stability classification}

The potential temperature is defined as the temperature that an air parcel would have if compressed or expanded adiabatically

to a standard pressure $P_{0}$. Formally [10],

$$
\theta(z)=T(z)\left[\frac{P_{0}}{P(z)}\right]^{\frac{R}{c_{p}}}
$$

where $T(z)$ is the air temperature $[K]$ at height $z[m], P_{0}$ is the air pressure $[h P a]$ at the surface level $\left(P_{0}=1022.1 h P a\right.$, mean air pressure during April 2015, in IJmuiden test site according to the Baltic and North Seas Climatology (BNSC)), $P(z)$ is the air pressure $[h P a], R \approx 287 \mathrm{JK}^{-1} \mathrm{~kg}^{-1}$ is the universal gas constant and $c_{p} \approx 1004 \mathrm{JK}^{-1} \mathrm{~kg}^{-1}$ is the specific heat capacity at a constant pressure $\left(\frac{R}{c_{p}} \approx 0.286\right)$. 
The atmospheric stability is classified as convective, neutral or stable based on the gradient of potential temperature, $\frac{d \theta}{d z}$, as

follows: $\frac{d \theta}{d z}<0$, convective; $\frac{d \theta}{d z}=0$, neutral; and, $\frac{d \theta}{d z}>0$, stable. In practice, the "zero" gradient was computed as the interval $\left[-3.89 \times 10^{-4}, 3.89 \times 10^{-4}\right][\mathrm{K} / \mathrm{m}]$, which is approximately the error-propagated gradient in response to the resolution $(1 \mathrm{hPa})$ of the pressure sensor Vaisala PTB210 conservatively increased by a factor 10 . Gradients were also calculated every 10 min using metmast temperature and pressure data at $21 \mathrm{~m}$ and $90 \mathrm{~m}$. When tackling atmosphere stability classification based on the wind-shear exponent, the neutral stability threshold interval was obtained by projecting the "zero" temperature gradient interval above through the straight line fit $(10.51 x+0.07)$ calculated for the scatter plot relating metmast wind-shear and potential temperature in Fig. 2. The wind-shear neutral-stability threshold interval thus obtained was $[0.066,0.074]$. At this point it is worth noting that although the central value (0.070) differs from previous values obtained in the literature (typically, 0.15) [5], [11], the neutral threshold interval is largely influenced by the quality of the fitting and fitting model used, which is still a matter of research. Values of wind-shear $\alpha>0.074$ classified the atmosphere as stable and $\alpha<0.066$ as convective (see Fig. 2 inset labels).

\section{DISCUSSION RESULTS}

Fig. 2 compares metmast-retrieved wind-shear exponent, $\alpha_{\text {mast }}$, against metmast-retrieved potential temperature gradient, $\frac{d \theta}{d z}$, over the whole campaign represented by 3924 measurements. Fig. 3 is the counterpart of Fig. 2 for the DWL. To intercompare these two data sets on a meaningful statistical sense, two steps of histogrammed filtering were applied: (i) gradient values, $\frac{d \theta}{d z}$, falling out of the $\mu \pm 1 \sigma$ interval, where $\mu$ is the mean value of the distribution and $\sigma$ is the standard deviation, were rejected as outliers. After that, 100 bins $\left(3.30 \times 10^{-04}\right.$ binwidth) were used to cover the entire range of $\frac{d \theta}{d z}$. (ii) For each $\frac{d \theta}{d z}$ bin, the distributions of metmast wind-shear values, $\alpha_{\text {mast }}$, and DWL values, $\alpha_{D W L}$, were histogrammed and limited to $\mu \pm 1 \sigma$ respective interval. Values out of the respective interval are marked as outlier and plotted as grey dots in Figs. 2-3, 5.

Comparing Fig. 2 analysis (2630 records after filtering), yielded a correlation coefficient, $\rho=0.80$, and a root-mean- 

DWL fitted-line slope, $\mathrm{m}=11.12$, is only $5.8 \%$ higher than that of metmast $(\mathrm{m}=10.51)$.

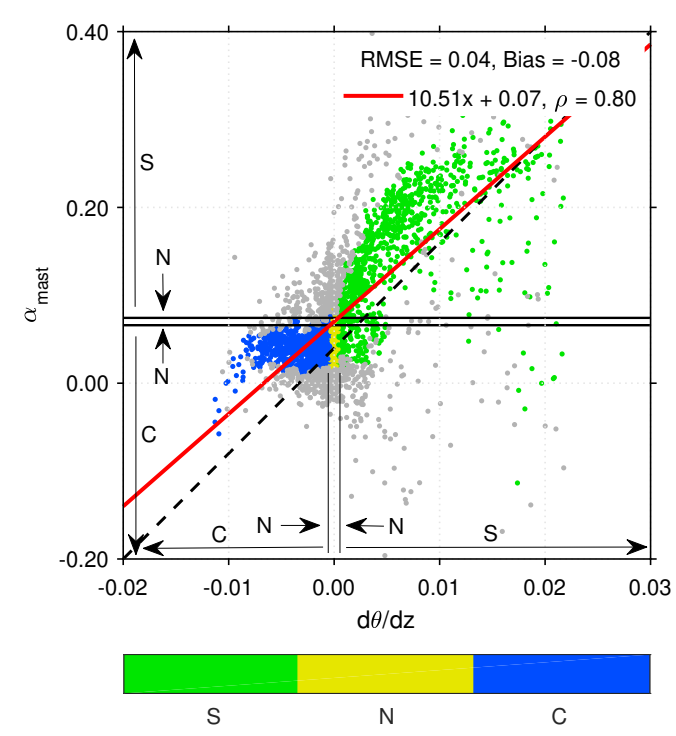

Fig. 2. Comparison between metmast wind-shear exponents, $\alpha_{m a s t}$, and metmast-retrieved potential temperature gradients, $\frac{d \theta}{d z}$.

squared error, RMSE = 0.04, which indicates a virtually linear relationship. Fig. 3 (2695 records after filtering) showed lower correlation, $\rho=0.67$, for the DWL. However, both, Fig. 2 and Fig. 3, straight line fits yield similar intercepts $(\mathrm{n}=0.07)$ and the

$\mathrm{S}$ is stable, $\mathrm{N}$ is neutral and $\mathrm{C}$, convective. (Gray dots) Rejected outliers. (Black dashed line) Ideal 1:1 line. (Red solid line)

Regression line. (Black solid lines) Wind-shear neutral-stability threshold interval.

Fig. 4 shows the percentage of cases for each atmospheric stability class according to stability indicators discussed. According to potential-temperature typing (Fig. 4 a) the atmosphere was predominantly convective in $48 \%$ of the cases, followed by the stable class, $37 \%$, and the neutral class, 15\%. Metmast wind-shear indicator, $\alpha_{\text {mast }}$ (Fig. 4 b), and DWL wind-shear, $\alpha_{D W L}$, (Fig. $4 \mathrm{c}$ ) classified the atmosphere as convective in $62 \%$ and $52 \%$ of the cases respectively, which represents an overestimation of $14 \%$ and $4 \%$. When the stable and neutral cases are added up $(\mathrm{S}+\mathrm{N})$ and compared against the convective one (C), the agreement between the metmast-retrieved potential-temperature gradients and DWL wind-shear-retrieved exponents is very good. Experiment also showed that results were largely influenced by the potential-temperature-gradient criterion used to define the neutral case interval. 


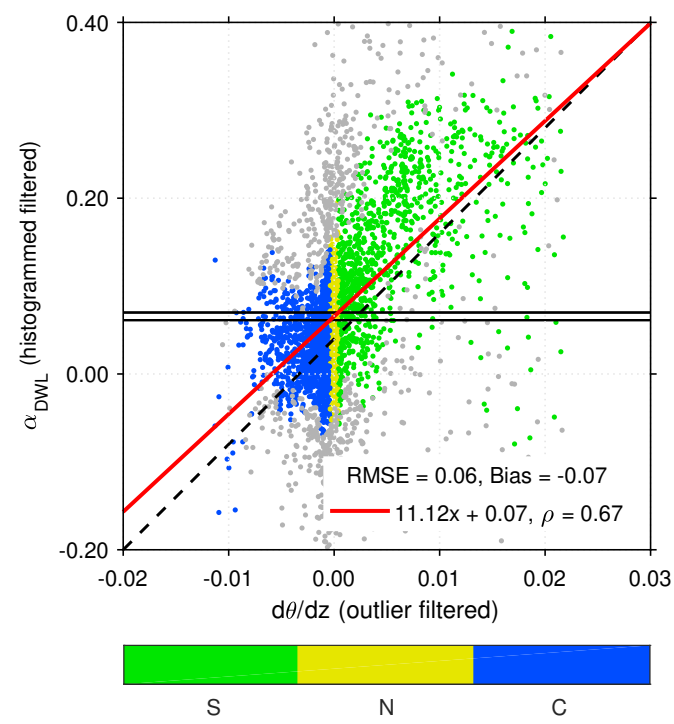

Fig. 3. Comparison between DWL wind-shear exponents, $\alpha_{D W L}$, and metmast-retrieved potential temperature gradients, $\frac{d \theta}{d z}$.

Same format as Fig. 2.
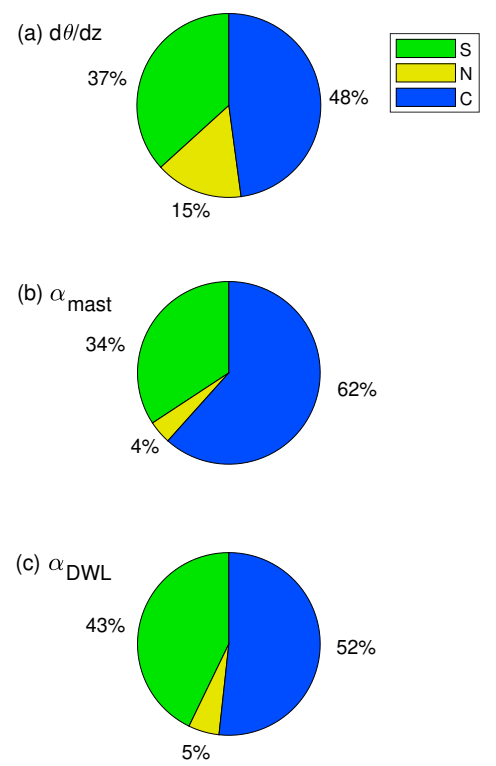

Fig. 4. Atmospheric stability classification based on the three different indicators studied. Labels $d \theta / d z, \alpha_{\operatorname{mast}}$ and $\alpha_{D W L}$ refer respectively to potential-temperature gradient indicator, metmast and DWL wind-shear indicators (total sample 2959 records).

$\mathrm{S}$ is stable, $\mathrm{N}$ is neutral and $\mathrm{C}$ is convective. 

records) the correlation coefficient was $\rho=0.88$ and the RMSE $=0.05$. The fitted line is close to the ideal 1:1 line and the null intercept is indicative of no bias. As expected, DWL wind-shear exponents became more scattered. We hypothesize that this scattering is consequence of the buoy motion-induced wind turbulence.

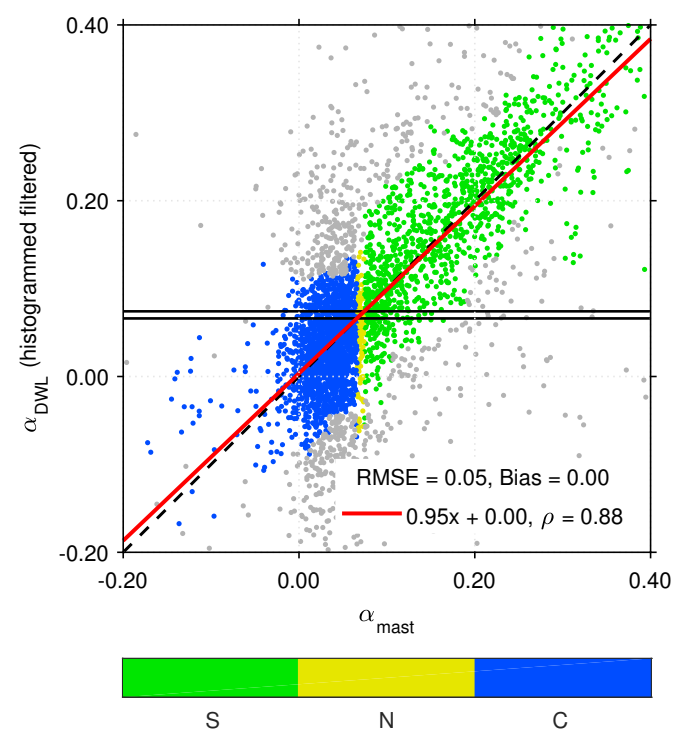

Fig. 5. Comparison between DWL and metmast wind-shear exponents, $\alpha_{D W L}$ and $\alpha_{m a s t}$, respectively. Same format as Fig. 2

\section{CONCLUSIONS}

The wind-shear parameter measured offshore by the lidar ( $\alpha_{D W L}$, Ijmuiden's campaign, 4319 records) was used as atmosphericstability typing indicator (stable, neutral, convective) and compared against two reference indicators: the metmast-retrieved potential temperature gradient $(d \theta / d z)$ and the metmast-retrieved wind shear $\left(\alpha_{\text {mast }}\right)$. When comparing metmast wind-shear

to the potential-temperature indicators $\left(\alpha_{\text {mast }}-\right.$ to $\left.-d \theta / d z\right)$ a correlation coefficient of 0.80 and a straight line fit with low bias (0.07) was obtained. This correlation reduced to 0.67 when comparing the lidar to the metmast $\left(\alpha_{D W L}-\right.$ to $\left.-d \theta / d z\right)$ on account of the motion-induced apparent wind turbulence. The lidar to the metmast-retrieved wind shear $\left(\alpha_{D W L}-\right.$ to $\left.-\alpha_{m a s t}\right)$ showed 
linear dependency $(\rho=0.88)$. Concerning stability typing, both the lidar and the metmast-derived wind-shear indicators overestimated the convective stability case (Fig. 4) in comparison to the potential-temperature reference indicator. A plausible explanation for that is that stability typing is largely sensitive on the wind-shear thresholds obtained for the neutral case, quality of the fitting and fitting model used.

[1] Global Wind Energy Council, “Global wind report 2018,” Tech. Rep., Global Wind Energy Council, Apr. 2019.

[2] Carbon Trust, "Carbon trust offshore wind accelerator roadmap for the commercial acceptance of floating lidar technology,” Tech. Rep., Carbon Trust, Oct. 2018.

[3] M.C. Holtslag, W.A.A.M. Bierbooms, and G.J.W. Van Bussel, "Estimating atmospheric stability from observations and correcting wind shear models accordingly," in Journal of Physics: Conference Series. IOP Publishing, 2014, vol. 555, p. 012052.

[4] C. L. Archer, B. A. Colle, D. L. Veron, F. Veron, and M. J. Sienkiewicz, "On the predominance of unstable atmospheric conditions in the marine boundary layer offshore of the u.s. northeastern coast," Journal of Geophysical Research: Atmospheres, vol. 121, no. 15, pp. 8869-8885, 2016.

[5] S. Wharton and J. Lundquist, "Assessing atmospheric stability and its impacts on rotor-disk wind characteristics at an onshore wind farm," Wind Energy, vol. 15, May 2012.

[6] A. Pẽnha, C. B. Hasager, B. Merete, R. J. Barthelmie, B. Ferhat, C. Jean-Pierre, E. Stefan, S. Frandsen, H. Michael, K. Ioanna, S. Larsen, M. Jakob, M. Torben, P. Mark, P. Sara, S. Ameya, S. David, S. Chris, and W. Rozenn, Remote Sensing for Wind Energy, DTU Wind Energy, Denmark, 2015. 
[7] M. A. Gutiérrez-Antuñano, J. Tiana-Alsina, A. Salcedo, and F. Rocadenbosch, "Estimation of the motion-induced horizontal-wind-speed standard deviation in an offshore doppler lidar,” Rem. Sens., vol. 10, no. 12, pp. 2037, Dec 2018.

[8] A. Salcedo, M. A. Gutierrez-Antunano, J. Tiana-Alsina, and F. Rocadenbosch, "Floating doppler wind lidar measurement of wind turbulence: A cluster analysis," in 2020 IEEE Int. Geo. Rem. Sens. Symp. (IGARSS). submitted 2019, IEEE.

[9] J. F. Manwell, J. G. McGowan, and A. L. Rogers, Wind energy explained: theory, design and application, John Wiley \& Sons, 2010.

[10] J. M. Wallace and P. V. Hobbs, Atmospheric science: an introductory survey, vol. 92, Elsevier, 2006.

[11] G. P. van den Berg, "Wind turbine power and sound in relation to atmospheric stability," Wind Energy, vol. 11, no. 2, pp. 151-169, 2008. 